\title{
Optimal TCSC Allocation via Chaotic Immune Symbiotic Organisms Search for Voltage Profile Improvement
}

\author{
Mohamad Khairuzzaman Mohamad Zamani ${ }^{1}$, Ismail Musirin ${ }^{2, *}$, Saiful Izwan Suliman ${ }^{3}$, Sharifah Azma Syed Mustaffa ${ }^{4}$, \\ Nur Zahirah Mohd $\mathrm{Ali}^{5}$, and Mohd Helmi Mansor ${ }^{6}$, Zulkiffli Abdul Hamid ${ }^{7}$ \\ ${ }^{1,2,3,5,7}$ Faculty of Electrical Engineering, Universiti Teknologi MARA, 40450 Shah Alam, Selangor, Malaysia \\ ${ }^{4,6}$ College of Engineering, Universiti Tenaga Nasional, Kajang, Selangor, Malaysia
}

\begin{abstract}
As the load demand in a power system increases, power system operators struggle to maintain the power system to be operated within its acceptable limits. If no mitigation actions are taken, a power system may suffer from voltage collapse, which in turn leads to blackout. Flexible AC Transmission System (FACTS) devices can be employed to help improve the voltage profile of the power system. This paper presents the implementation of Chaotic Immune Symbiotic Organism Search (CISOS) optimization technique to solve optimal Thyristor Controlled Series Compensator (TCSC) in a power system for voltage profile improvement. Validation process are conducted on IEEE 26-bus RTS resulting in the capability of CISOS in solving the allocation problem with a better voltage profile. Comparative studies conducted with respect to Particle Swarm Optimization (PSO) and Evolutionary Programming (EP) has revealed the superiority of CISOS over PSO and EP in solving the optimal allocation problem by producing optimal solution with a better voltage profile. The results and information obtained from this study can help power system operator in terms of optimal compensation in power system as well as improving the operation of a power system.
\end{abstract}

\section{Introduction}

In power system operation, it is crucial for the voltage profile of the power system to be maintained within the acceptable level. As the load demand in a power system increases, power system operators struggle to keep the voltage profile within its appropriate level. If such event is not mitigated, it may later cause reduction in voltage value, which later leads to voltage collapse. In order to mitigate such event from occurring, several mitigation steps can be implemented by power system operators.

On of the mitigation step which can be implemented is to install compensating devices on a power system. Flexible AC Transmission System (FACTS) devices are one of available compensating devices which can be used. Many previous studies have implemented FACTS devices for power system compensation and manages to provide improvement to the power system operation. FACTS devices have been implemented to solve various power system operation problems related to voltage stability improvement, voltage profile improvement, power loss minimization as well as maximization of power transfer capability.

Berrouk et. al. [1] has conducted a study on the implementation of FACTS devices to improve the voltage profile of the IEEE 30-bus power system. In the study, 2 FACTS devices have been implemented in order to provide improvement to the voltage level at selected buses which are Static VAr Compensator (SVC) and
Static Compensator (STATCOM). The results obtained from the study reveals that both devices are able to provide voltage profile improvement to a power system, not only to the bus which the devices are installed, but throughout the system, hence improving the operation of the power system.

In different study, Bavithra et. al. [2] has implemented Particle Swarm optimization (PSO) technique to optimally determine the allocation of FACTS devices in a 6-bus power system with the objective to maximize the available transfer capability of the system. The study reveals that FACTS devices are capable of enhancing the active power transfer capability of a power system, which would allow further loading on the power system.

In the effort to achieve optimality in the allocation of FACTS devices in a power system, many researchers have implemented metaheuristics-based optimization technique to solve various optimization problems. Bhattacharyya et. al. [3] has implemented Differential Evolution (DE) technique to optimal planning of FACTS devices installation to increase the loadability of IEEE 30-bus system. Implementation of Evolutionary Programming (EP) has been conducted by Salim et. al. [4] to solve optimal allocation of FACTS devices in IEEE 30-bus system to improve the voltage profile of the system as well as minimizing total power loss in the system. 
Aside of PSO, DE and EP, various researchers have implemented other optimization techniques which is able to provide good optimal solutions in solving optimal FACTS devices allocation problem in a power system. In [5], the authors have implemented Flower pollination Algorithm (FPA) to determine the optimal allocation of SVC to minimize total power loss in a power system. Another study to minimize total power loss in a power system has been conducted by Taleb et. al. [6] through optimal allocation of Thyristor Controlled Series Compensator (TCSC) in a power system using Cuckoo Search Algorithm (CSA). Gitizadeh [7] has proposed the implementation of modified version of Simulated Annealing (SA) optimization technique to solve optimal FACTS devices installation for power system congestion alleviation. Another study to improve the voltage stability of a power system as well as reducing total power loss by optimal allocation of FACTS devices using Harmony Search Algorithm (HSA) and Genetic Algorithm (GA) has been conducted by authors in [8].

Although the existing optimization techniques are able to solve the optimal FACTS devices allocation problem, several studies have reported drawbacks which hinders the optimization techniques from providing highquality solution. Authors in [9] has reported that conventional optimization techniques such as Linear Programming (LP), Non-Linear Programming (NLP) and Mixed-Integer Non-Linear Programming are unable to deal with an optimization problem which are nonconvex and discontinuous in nature. Dubey et. al. [10] has reported that GA suffers from problems of divergence and tendency to stuck at local optima despite of being a good optimization technique. Authors in [10] also reported that SA technique is computationally intensive and it is very sensitive to its control parameter. Authors in [11] has presented that heuristic optimization techniques generally suffers from slow convergence.

This paper presents the implementation of Chaotic Immune Symbiotic Organisms Search (CISOS) optimization technique to solve optimal TCSC allocation problem with the aim to improve the voltage profile of the power system. CISOS was inspired by the improvement of the original Symbiotic Organisms Search (SOS) optimization technique developed by Cheng and Prayogo [12]. SOS has been implemented to solve various optimization problems such as economic dispatch [13], optimal power flow [14] and optimal FACTS device installation [15][16]. Effectiveness of SOS is further improved through modification which inspires the development of SOS-based optimization techniques such as Chaotic SOS [17], Chaos Embedded SOS [18], Adaptive SOS [19], as well as CISOS [20]. The optimization process will be tested on IEEE 26-bus Reliability Test System (RTS) and the observed results will be the value of total voltage deviation index (TVDI), which reflects the voltage profile of the system. Comparative studies are also conducted with respect to PSO and EP in terms of producing optimal solution which would yield better voltage profile improvement.

\section{Methodology}

To solve the problems mentioned in the previous section, CISOS optimization technique is implemented to determine the optimal allocation of TCSC in a power system. The detail about the optimization problem are explained as in section 2.1 while section 2.2 briefly describe the implementation of the proposed CISOS optimization technique for optimal TCSC allocation problem.

\subsection{Problem Formulation}

In this study, CISOS is implemented to determine the optimal location and sizing of TCSC to be installed in the test system with the aim to improve the voltage profile of the system by minimizing the total voltage deviation index value. Hence, the objective function of the optimization can be mathematically expressed as:

$$
F=\min (T V D I)
$$

where TVDI is the total voltage deviation index value. TVDI is defined as the total deviation value of a voltage value at a certain bus with respect to the reference voltage value at the same bus. The reference voltage value for a load bus is defined to be 1.00 p.u. while the reference voltage value for swing and generator buses are defined to be the actual voltage setpoint value for the generators prior to the installation of TCSC. The fitness function of the optimization process is the TVDI value, and it can be expressed mathematically as:

$$
T V D I=\sum_{k=1}^{n_{b u s}}\left(\frac{V_{r e f, k}-V_{k}}{V_{r e f, k}}\right)^{2}
$$

where $V_{k}$ is the $k^{\text {th }}$ bus voltage and $V_{r e f, k}$ is the reference voltage value for $k^{\text {th }}$ bus.

A Thyristor Controlled Series Compensator (TCSC) is capable of providing compensation by changing the reactance of a transmission line. The change in reactance of transmission lines will affect the impedance of the transmission lines. Figure 1 illustrates the single line diagram of a TCSC connected in series with a transmission line connecting 2 buses in a power system.

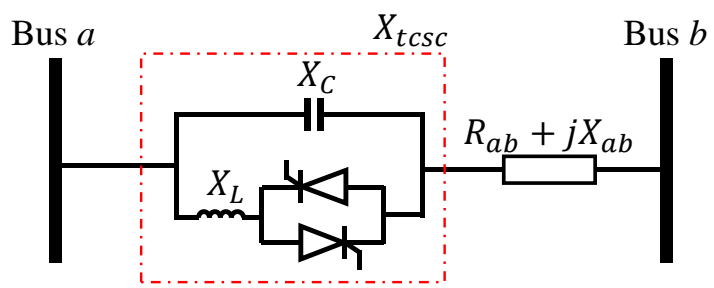

Fig. 1. Single line diagram of TCSC connected to 2 buses

In this paper, sizing of TCSC is referred to the compensation ratio of TCSC, which can be defined as 
the ratio of the reactance of TCSC to the reactance of an uncompensated transmission line, which can be expressed as:

$$
\gamma_{T C S C}=\frac{X_{T C S C}}{X_{a b}}
$$

After a TCSC is installed to a transmission line, total reactance of the compensated transmission line can be defined as the sum of reactance of TCSC as well as the uncompensated transmission line. The total reactance of the compensated line can be expressed as in (4).

$$
X_{a b}^{\prime}=X_{a b}+X_{T C S C}
$$

By rearranging (3) and substituting $X_{T C S C}$ in (4), $X_{a b}^{\prime}$ can be expressed in terms of $X_{a b}$ as:

$$
X_{a b}^{\prime}=\left(1+\gamma_{T C S C}\right) X_{a b}
$$

During the optimisation process, the value of TCSC compensation ratio is limited to its minimum and maximum limit value. The minimum value of $\gamma_{T C S C}$ is set to -0.8 p.u. while the maximum value of $\gamma_{T C S C}$ is set at 0.2 p.u. The limitation of $\gamma_{T C S C}$ can be mathematically expressed as in (6).

$$
\gamma_{T C S C}^{\min } \leq \gamma_{T C S C} \leq \gamma_{T C S C}^{\max }
$$

\subsection{Chaotic Immune Symbiotic Organisms Search (CISOS) for TCSC Installation}

In order to determine the optimal TCSC compensation ratio value as well as the location of TCSC to be installed in the tests, this paper implements an optimization technique known Chaotic Immune Symbiotic Organisms Search (CISOS) optimization technique to solve the problem. CISOS is proposed by Mohamad Zamani et. al. [20]. The proposed optimization technique was inspired by authors in [12] with an improvement in order to improve the searching capability of the original SOS developed by [12].

The detail optimization process of CISOS can be referred at [20]. The optimization process to determine the optimal allocation of TCSC in the test system is briefly described as follows:

Step 1: Initialization. At this stage, a set of possible $\gamma_{T C S C}$ as well as its location was generated by using random number. The generation of the random number is continued until the entire pool of organisms has been filled with $N_{\text {org }}$ organisms. $N_{\text {org }}$ is the number of organisms in the initial ecosystem (pool of organisms). During initialization process, several parameters will also be initialized such as best organism, chaotic search space radius value, maximum iteration of Chaotic Local Search as well as maximum iteration for the optimization process.
Step 2: Cloning. After the entire pool of organisms has been filled with the possible solutions, the ecosystem is then cloned with $N_{\text {clone }}$ copies of pool of organisms, hence creating a resulting ecosystem larger than the initial (current) ecosystem. $N_{\text {clone }}$ is defined as the number of clones.

Step 3: Symbiosis Relationship Phases. Then, the resultant ecosystem is then subjected to the symbiotic relationship phases. In this stage, the ecosystem will be subjected to 3 symbiosis relationship phases, namely mutualism, commensalism and parasitism. At the end of the symbiosis relationship phases, the ecosystem is modified based on the results of the subjection of the ecosystem to the symbiotic relationships.

Step 4: Best Organism Identification. After the symbiosis relationship phases has been completed, then, the algorithm will determine the best organism among the organisms in the ecosystem. In this study, the best organism is defined as the organism which has the lower fitness value or TVDI value. The chosen best organism will replace the older best organism in case of the latter best organism possess a better fitness value.

Step 5: Symbiosis Relationship Termination Test. The algorithm will test to determine whether all the organisms in the ecosystem has been subjected to the symbiosis relationship phases. If not all organisms have been subjected to the symbiosis relationship phases, then the optimization process will continue back to step 3 . Otherwise, the optimization process continues to step 6 .

Step 6: Elimination. The current ecosystem size will be reduced by choosing $N_{\text {org }}$ best organisms in the ecosystem. The chosen organisms will have a better fitness value to be carried forward in the optimization process while discarding the remaining possible solutions.

Step 7: Chaotic Local Search (CLS). A local search is conducted around the best organism in the effort to find a better possible solution. The chaotic local search will employ the usage of Piecewise Local Chaotic Map (PLCM) chaotic mapping to generate a random possible solution around the best organism. The fitness solution will be computed and compared with the fitness value of the best organism. In the event of CLS is unable to find a better solution, the process continues to the update using PLCM mapping until a better solution is found or the CLS iteration counter has reached its maximum. Otherwise, should the CLS manage to find a better solution, the solution will replace the best organism, CLS is terminated, the chaotic search space radius is reduced, and the optimization process continues.

Step 8: Convergence Test. If the optimization technique has reached its maximum iteration value, the optimization process will be terminated. However, if the iteration counter hasn't reached its maximum value, the iteration counter will be updated, and the optimization process continues to step 2 . 


\section{Results and Discussions}

In order to test the effectiveness of the optimization technique in solving the optimal TCSC allocation problem, the optimization process is tested on IEEE 26bus reliability test system (RTS). Figure 2 illustrates the single line diagram of the IEEE 26-bus RTS. The optimization parameters of CISOS are listed as in Table 1.

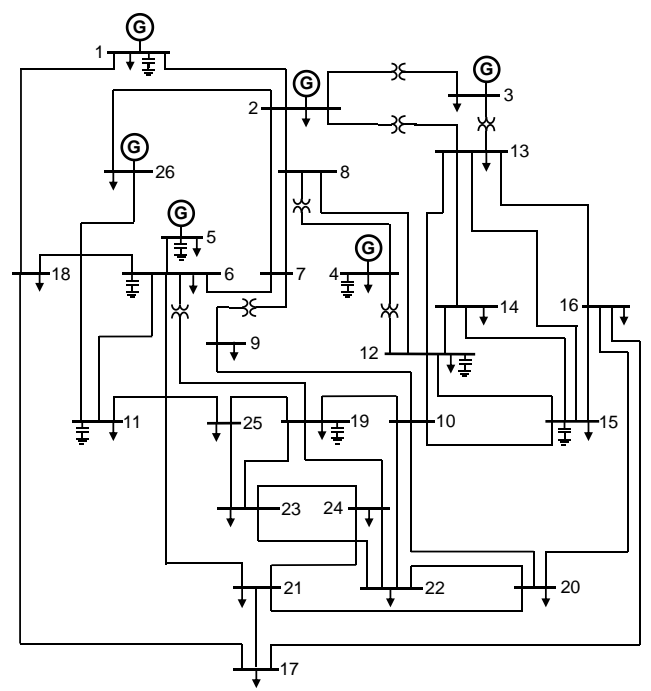

Fig. 2. Single line diagram of IEEE 26-bus RTS

Table 1. Optimization parameter of CISOS.

\begin{tabular}{|l|c|l|}
\hline Number of initial organisms, $N_{\text {org }}$ & $:$ & 20 \\
\hline Number of clones, $N_{\text {clone }}$ & $:$ & 10 \\
\hline Number of TCSC installed & $:$ & 3 \\
\hline CLS control parameter & $:$ & 0.4 \\
\hline Maximum CISOS iteration & $:$ & 100 \\
\hline Maximum CLS iteration & $:$ & 50 \\
\hline
\end{tabular}

Table 2. Results obtained from the optimization process.

\begin{tabular}{|c|c|c|}
\hline Parameter & Technique & TVDI \\
\hline Pre-optimized & - & 0.00483 \\
\hline \multirow{3}{*}{ Best post-optimized } & CISOS & 0.00220 \\
\cline { 2 - 3 } & PSO & 0.00244 \\
\cline { 2 - 3 } & EP & 0.00321 \\
\hline \multirow{3}{*}{ Worst post-optimized } & CISOS & 0.00223 \\
\cline { 2 - 3 } & PSO & 0.00297 \\
\cline { 2 - 3 } & $\mathrm{EP}$ & 0.00405 \\
\hline \multirow{3}{*}{ Average post-optimized } & $\mathrm{CISOS}$ & 0.00220 \\
\cline { 2 - 3 } & $\mathrm{PSO}$ & 0.00270 \\
\cline { 2 - 3 } & $\mathrm{EP}$ & 0.00356 \\
\hline
\end{tabular}

In order to perform comparative study to determine the superiority of CISOS, the same optimization problem is solved using other optimization techniques such as Particle Swarm Optimization (PSO) and Evolutionary Programming (EP). For each optimization technique, the optimization process will be conducted for 10 times to observe the variation of results produced. In the optimization process, the test system is simulated at base case condition in the IEEE 26-bus RTS. The results obtained from the optimization process is tabulated as in Table 2.

From the results obtained and tabulated in Table 2, it is clear that the proposed CISOS optimization technique is able to solve the optimal TCSC allocation problem which has been indicated by the reduction of TVDI value in the best post-optimized, worst post-optimized and average post-optimized results compared to the preoptimized TVDI value. The reduction of TVDI value indicates the reduction of voltage deviation, which reflects to the improvement of the voltage profile in the power system. The best optimal solution produced by CISOS has managed to provide $54.45 \%$ improvement in voltage profile indicated by the reduction of TVDI value from 0.00483 down to 0.00220 .

Worst post-optimized results are defined as the result with the highest TVDI value among the 10 optimization runs conducted. Average post-optimized results are the average of the fitness values obtained from all the optimization process conducted. In terms of CISOS, the worst post-optimized and the average post-optimized fitness values are 0.00223 and 0.00220 , which are lesser than the pre-optimized fitness value. These values indicate that the proposed CISOS optimization technique has managed to solve the optimization problem.

The results of optimal TCSC allocation in IEEE 26bus RTS by using PSO and EP are tabulated in Table 2 . In general, both PSO and EP managed to solve the allocation problem indicated by the reduction of TVDI values indicated by the best post-optimized, worst postoptimized and average post-optimized fitness values. CISOS has proven its superiority over PSO and EP by yielding better results compared to PSO and EP. CISOS has produced lower fitness value compared to the fitness value of best post-optimized results in PSO and EP. The superiority is further highlighted by the minimal TVDI value produced by CISOS in terms of worst postoptimized and average post-optimised TVDI values of PSO and EP. These results exhibit that CISOS is capable of solving the optimal TCSC allocation problem for better voltage profile improvement as compared to PSO and EP.

\section{Conclusion}

This paper presents the implementation of Chaotic Immune Symbiotic Organisms Search (CISOS) optimization technique for solving optimal TCSC allocation problem for voltage profile improvement in power system. In order to achieve a good voltage profile, compensation scheme as well as the optimality of the compensation should be taken into consideration. From the study, the implementation of the proposed CISOS optimization technique has managed to solve the optimization problem with a good optimal solution. The compensation ratio and location of TCSC installation produced by CISOS has improved the voltage profile of IEEE 26-bus RTS which has been indicated by the reduction of TVDI value. Through comparative studies, it can be observed that CISOS has yielded its superiority over PSO and EP by producing better results in terms of 
post-optimized fitness value compared to the values produced by PSO and EP. The results obtained from this study can be implemented by power system operators in order to increase the optimality of the power system, hence improving the power system operation.

\section{Acknowledgement}

The authors would like to acknowledge The Institute of Research Management and Innovation (IRMI), Universiti Teknologi MARA (UiTM), Shah Alam, Selangor, Malaysia for the support of this research. This research is supported by IRMI under Research Entity Initiative (REI) with project code: 600-IRMI/REI 5/3 (011/2018).

\section{References}

1. F. Berrouk, B. Ali Rachedi, A. Lemzadmi, K. Bounaya, and H. Zeghache, Applications of shunt FACTS controller for voltage stability improvment, 2014 International Conference on Electrical Sciences and Technologies in Maghreb (CISTEM), $1-6,(2014)$.

2. K. Bavithra, S. Charles Raja, and P. Venkatesh, Optimal Setting of FACTS Devices using Particle Swarm Optimization for ATC Enhancement in Deregulated Power System, IFAC-PapersOnLine, 49, 450-455, (2016).

3. B. Bhattacharyya and S. K. Goswami, Optimal Planning for the Placement of FACTS Devices by Differential Evolution Technique for the Increased Loadabilty of a Power System, 2012 Asia-Pacific Power and Energy Engineering Conference, 1-4, (2012).

4. N. A. B. Salim and J. Maika, Optimal allocation of FACTS device to improve voltage profile and power loss using evolutionary programming technique, 2016 IEEE Region 10 Conference (TENCON), 1208-1215, (2016).

5. B. Sravan Kumar, M. Suryakalavathi, and G. V. Nagesh Kumar, Optimal power flow with static VAR compensator based on flower pollination algorithm to minimize real power losses, 2015 Conference on Power, Control, Communication and Computational Technologies for Sustainable Growth (PCCCTSG), 112-116, (2015).

6. M. Taleb, A. Salem, A. Ayman, and M. A. Azma, Optimal allocation of TCSC using adaptive cuckoo search algorithm, 2016 Eighteenth International Middle East Power Systems Conference (MEPCON), 387-391, (2016).

7. M. Gitizadeh, A modified simulated annealing approach to congestion alleviation in a power system using FACTS devices, 45th International Universities Power Engineering Conference UPEC2010, 1-6, (2010).

8. A. Parizad, A. Khazali, and M. Kalantar, Application of HSA and GA in optimal placement of FACTS devices considering voltage stability and losses, 2009 International Conference on Electric Power and Energy Conversion Systems, (EPECS),
$1-7,(2009)$.

9. S. Ranganathan, M. Surya Kalavathi, and C. Asir Rajan C., Self-adaptive firefly algorithm based multi-objectives for multi-type FACTS placement, IET Generation, Transmission \& Distribution, 10, 2576-2584, (2016).

10. R. Dubey, S. Dixit, and G. Agnihotri, Optimal Placement of Shunt Facts Devices Using Heuristic Optimization Techniques: An Overview, 2014 Fourth International Conference on Communication Systems and Network Technologies, 518-523, (2014).

11. S. Dutta, P. K. K. Roy, and D. Nandi, Optimal location of TCSC and TCPS using hybrid DE/CRO algorithm, Michael Faraday IET International Summit, 170-175, (2015).

12. M.-Y. Cheng and D. Prayogo, Symbiotic Organisms Search: A new metaheuristic optimization algorithm, Computers \& Structures, 139, 98-112, (2014).

13. U. Guvenc, S. Duman, M. K. Dosoglu, H. T. Kahraman, Y. Sonmez, and C. Yilmaz, Application of Symbiotic Organisms Search Algorithm to solve various economic load dispatch problems, 2016 International Symposium on INnovations in Intelligent SysTems and Applications (INISTA), 1-7, (2016).

14. D. Prasad and V. Mukherjee, A novel symbiotic organisms search algorithm for optimal power flow of power system with FACTS devices, Engineering Science and Technology, an International Journal, 19, 79-89, (2016).

15. M. K. Mohamad Zamani, I. Musirin, and S. I. Suliman, Symbiotic Organisms Search Technique for SVC Installation in Voltage Control, Indonesian Journal of Electrical Engineering and Computer Science, 6, 318-329, (2017).

16. M. K. Mohamad Zamani, I. Musirin, S. A. Syed Mustaffa, and S. I. Suliman, Optimal SVC allocation via symbiotic organisms search for voltage security improvement, TELKOMNIKA, 17, 1267-1274, (2019).

17. M. Abdullahi, M. A. Ngadi, and S. I. Dishing, Chaotic symbiotic organisms search for task scheduling optimization on cloud computing environment, 2017 6th ICT International Student Project Conference (ICT-ISPC), 1-4, (2017).

18. S. Saha and V. Mukherjee, Optimal placement and sizing of DGs in RDS using chaos embedded SOS algorithm, IET Generation, Transmission \& Distribution, 10, 3671-3680, (2016).

19. G. G. Tejani, V. J. Savsani, and V. K. Patel, Adaptive symbiotic organisms search (SOS) algorithm for structural design optimization, Journal of Computational Design and Engineering, 3, 226249, (2016).

20. M. K. Mohamad Zamani, I. Musirin, S. I. Suliman, and S. A. Syed Mustaffa, Chaotic Immune Symbiotic Organisms Search for solving optimisation problem, International Journal of Engineering \& Technology, 7, 73-79, (2018). 